\title{
Application of Stochastic Linear Programming in the Solution of a Transportation Problem
}

\author{
Ahmed M. Shams ${ }^{1}$, El Sayed Sorour ${ }^{2}$, Elewa Roshdy ${ }^{3}$ and Sameh Farahat ${ }^{4}$
}

\begin{abstract}
:
Stochastic Linear Programming (SLP), has a great importance due to its various applications in real life. In particular, the two-stage SLP or, sometimes, recourse programming. We used the two-stage SLP to describe the common transportation problem in case of random levels of supplies and demands. The randomness in the supplies and demands levels gives more realistic description of the problem. The random parameters in such problems may be continuously or discretely distributed. Indeed, there are many algorithms used to solve such problems in both continuous and discrete cases. The L-shape algorithm is the most commonly used in the discrete case two-stage SLP, but the problem in this method is the need for large computer memory to perform the iterations in such problems which have large numbers of decision variables. We give an example of such transportation problems and carry out using two distinct designs for the problem. One, using a subcontract with very high cost as penalty for demands not met. The other, using a virtual extra-demand at each supplier and these extra amounts act like supplying the over-demands. In each design, we calculate the total expected cost once using the expectation of each random variable, and another using the most likely realization of each random variable. We perform analysis of variance ANOVA to compare the four treatments from statistical point of view. Detailed results were illustrated for each design. All computations were performed with Matlab R2014a and MS-Excel2014.
\end{abstract}

Keywords: Stochastic Linear programming (SLP), transportation problem, Stochastic Integer Programming, Analysis of variance, Test hypothesis, Pairwise comparison.

\footnotetext{
${ }^{1}$ Eng. Mathematics Department, Military Technical Collage-Cairo, Egypt, a.shams44mtc@gmail.com.

${ }^{2}$ Prof. Dr. in Eng. Mathematics Department, Military Technical Collage-Cairo, Egypt.

${ }^{3}$ Assoc. Prof. in Eng. Mathematics Department, Military Technical Collage-Cairo, Egypt.

${ }^{4}$ Dr. in Military Technical Research Center-Cairo, Egypt.
} 


\section{1- Introduction:}

Mathematical programming problems involving uncertain parameters, for which stochastic models are available, occurs in almost all areas of science and engineering, from telecommunication and medicine to finance. In particular, linear programs have been studied in many aspects during the last 50 years. They have shown to be appropriate models for a wide variety of practical problems and, at the same time, they became numerically tractable even for very large scale instances. The fundamental assumption for linear programming, that the problem entries-except for the decision variables-be known fixed data, does often happen not to hold, but usually they are random data. In general, a LPP of the form:

$$
\begin{aligned}
\min z & =c^{T} x \\
\text { S.t. } \quad A x & =b \\
x & \geq 0
\end{aligned}
$$

Where $x \in X \subset R^{n}, c \in R^{n}, b \in R^{m}, X$ is convex polyhedral set and $A$ is $m \times n$ matrix representing the decision variables and the problem data, with some or all $(A, b, c)$ are random data with known (joint) probability distribution is called Stochastic Linear Programming Problem [2]. Stochastic models gained that importance due to:

1- Randomness is needed to obtain a correct evaluation of the future income and objectives.

2- Flexibility only has value (and meaning) in a setting of randomness.

3- Only by explicitly evaluating future decisions, can decisions containing flexibility be correctly valued.

The basic assumption is that the probability distribution of the random entries does not depend on the decision vector $x$. Note that the presence of uncertainty affects both feasibility and optimality. To understand this, consider the following four variables LPP[4]:

$$
\begin{array}{cc} 
& \min -x_{2} \\
\text { s.t. } & a_{1} x_{1}+a_{2} x_{2}+x_{3}=2 \\
& a_{1} x_{1}+a_{2} x_{2}+x_{4}=2 \\
& -1 \leq x_{1} \leq 1 \\
& x_{2}, x_{3}, x_{4} \geq 0
\end{array}
$$

Suppose that the coefficients of $x_{1}$ and $x_{2}$ are not known with certainty, and all what is known is their joint probability distribution $\left(a_{1}, a_{2}\right)$ : 


$$
=\left\{\begin{array}{c}
\left(1, \frac{3}{4}\right) \text { with probability } \frac{1}{2} \\
\left(-3, \frac{5}{4}\right) \text { with probability } \frac{1}{2}
\end{array}\right.
$$

In this case, $E\left[a_{1}\right]=-1$ and $E\left[a_{2}\right]=1$, so if we replaced $\left(a_{1}, a_{2}\right)$ with their expected values and examined its solution $\left(x_{1}, x_{2}, x_{3}, x_{4}\right)=(1,3,0,0)$ under uncertainty, so that the constraints are equally likely to be either:

$$
\begin{aligned}
& x_{1}+\frac{3}{4} x_{2}+x_{3}=2 \\
& x_{1}+\frac{3}{4} x_{2}+x_{4}=2
\end{aligned}
$$

Or

$$
\begin{aligned}
& -3 x_{1}+\frac{5}{4} x_{2}+x_{3}=2 \\
& -3 x_{1}+\frac{5}{4} x_{2}+x_{4}=2
\end{aligned}
$$

We will find that the vector $(1,3,0,0)$ does not satisfy either of these constraints, thus is infeasible under uncertainty. That is, the solution of SLP problem in which the expected values are used to represent the random data is not always feasible.

Another approach is the wait-and-see analysis (sometimes referred to as scenario analysis or what-if analysis). This approach mimics the process of delaying all the decisions until all uncertain data are realized. As a result, we have a set of deterministic LPs correspond to each possible realization of the random data. This yields a set of decision vectors one for each LP, but none of these may be worthwhile. In the previous example, the solution associated with $\left(a_{1}, a_{2}\right)=\left(1, \frac{3}{4}\right)$ is $(-1,4,0,0)$ while the solution associated with $\left(a_{1}, a_{2}\right)=\left(-3, \frac{5}{4}\right)$ is $(1,4,0,0)$. Neither of these solutions is feasible with respect to the alternate case. From this results, the decision maker should consider the consequences of future infeasibility within the model under uncertainity. In the stochastic programming literature, two approaches are widely studied: one is based on modeling future recourse (response) and another restricts the probability of infeasibility (typically equivalent to system failures) to be no greater than a prespecified threshold. The first approach yields the so-called recourse problems (or two-stage problem), and the second approach yields problems with probabilistic (or chance) constraints. We focus on the recourse (two-stage) SLP. The paper is arranged as follows: in part 2 we clarify the concept of SLP with recourse, in part 3 we give an example of transportation problem formulated as two-stage SLP with two designs and a full statistical analysis of all outcomes.

\section{2- The two-stage SLP:}

A simple formulation of the Two-stage SLP is given, in which the randomness is introduced in the objective function of the recourse problem. 


$$
\begin{aligned}
& \min c^{T} x+\mathcal{Q}(x) \\
& \text { S.t. } A x=b, \quad x \geq 0 .
\end{aligned}
$$

Where the expected recourse cost

$$
\mathcal{Q}(x)=E_{\omega}(Q(x, \omega))
$$

and

$$
Q(x, \omega)=\min \left\{q^{T}(\omega) y \mid W(\omega) y=h(\omega)-T(\omega) x, y \geq 0\right\}
$$

Where $x \in X \subset \mathbb{R}^{n_{0}}, \quad y \in Y \subset \mathbb{R}^{n_{1}}, \quad b \in \mathbb{R}^{m_{0}}, \quad h \in \mathbb{R}^{m_{1}}, \omega \in \Xi \subset \mathbb{R}^{k}$. The function $Q(x, \omega)$ is called the recourse function and therefore the function $\mathcal{Q}(x)$ is called the expected recourse function. [3]

This representation clearly illustrates the sequence of events in the recourse problem. First-stage decisions $x$ are taken in the presence of uncertainty about future realizations of $\omega$. In the second stage, the actual value of $\omega$ becomes known and some corrective actions or recourse decisions $y$ can be taken. First-stage decisions are, however, chosen by taking their future effects into account. These future effects are measured by the value function or recourse function, $Q(x)$, which computes the expected value of taking decision $x$. If $c^{T} x$ and $\mathcal{Q}(x)$ are both convex in $x \forall \omega \in \Xi$, and $X$ is convex set then the SLP is convex. If ,for some $x$ and $\omega \in \Xi$, the second stage problem is infeasible, then by definition $Q(x, \omega)=+\infty$. Also, if the second stage problem is unbounded from below then $Q(x, \omega)=-\infty$.

For any given $\omega$ the function $Q(., \omega)$ is convex. Moreover, $Q(., \omega)$ is polyhedral if the set $\left\{\pi: W^{T} \pi \leq q\right\}$ of feasible solutions of the dual problem of the second stage problem is nonempty, and the second stage problem itself is feasible for at least one $x$.

A two stage SLP in which the $m_{1} \times n_{1}$ matrix $W(\omega)=W$ (deterministic) is called twostage SLP with fixed recourse. If this fixed matrix $W$ satisfies [the system $W y=\chi, y \geq$ 0 has a solution for every $\chi \in R^{m_{1}}$, then the problem is complete fixed recourse.

For the full proof of these properties, see [3 Chapter 2]. The objective function in (3) contains a deterministic term $c^{T} x$ and the expectation of the second-stage objective $q(\omega)^{T} y(\omega)$ taken over all realizations of the random event $\omega$. In case of $\omega$ is a discrete random variable, which is an important class of random variables, and is widely used in applications, then, if $\omega=\omega_{k}, k=1, \ldots K$ with respective probabilities $p_{k}$, represent $K$ realizations of the random variable $\omega$, which is discontinuous measure of noisy data or sampling with size $K$ of a continuous distribution, then we may write the whole large scale linear program:

$$
\min z=c^{T} x+\sum_{k=1}^{K} p_{k} Q\left(x, \omega_{k}\right)
$$

$$
\begin{gathered}
\text { s.t. } \quad T_{k} x+W_{k} y_{k}=h_{k} \quad k=1, \ldots K \\
A x=b
\end{gathered}
$$




$$
x \geq 0, \quad y_{k} \geq 0 \quad k=1, \ldots K
$$

Of course, if for at least one $k \in\{1, \ldots, K\}$ the system $T_{k} x+W_{k} y_{k}=h_{k}, y_{k} \geq 0$ has no solution, i.e. the corresponding second-stage program is infeasible, then its optimal value is $+\infty$, then the expected recourse $E[Q(x, \omega)]=\sum_{1}^{K} p_{k} Q\left(x, \omega_{k}\right)$ equal $+\infty$ if at least one of $Q\left(x, \omega_{k}\right)$ is $+\infty$. The proof of the optimality of problem (3) is given in [3].

In many situations, certain design variables can have only integer values. Typical example in some cases of transportation problem in which the decision variables must have integer values like people, cars and so on. One can say it is possible to round off the solution obtained from any solution algorithm to the nearest integer. However, in many cases, it is very difficult to round off the solution without violating any of the constraints. Frequently, the rounding of certain variables requires substantial changes in the values of some other variables to satisfy all the constraints. Further, the round-off solution may give a value of the objective function that is very far from the original optimum value. All these difficulties can be avoided if the program is posed and solved as an integerprogramming problem.

When all the variables are constrained to take only integer values in a programming problem, it is called an all-integer programming problem. When some variables only are restricted to take integer values, the programming problem is called a mixed-integer programming problem.[5]

The interfering of integrality in the two-stage SLP is simply adding a condition to restrict the desired decision variables to the set of integer numbers:

$$
\begin{aligned}
& \min c^{T} x+E_{\omega}\left[\left\{\min q^{T} y \mid T x+W y=h, y \geq 0\right\}\right] \\
& \text { s.t } A x=b, x \geq 0
\end{aligned}
$$

Here, $x \in X$ and $y \in Y$ such that $X$ and/or $Y$ are subsets of $\mathbb{Z}^{+}$. In general, the expected recourse cost in this case is nonconvex and discontinuous, and the second-stage feasibility set $K_{2}$ is nonconvex, for more discussion of feasibility and optimality conditions,[2].

Computation in stochastic programs with recourse has focused on two-stage problems with finite numbers of realizations. The general model is to choose some initial decision that minimizes current costs plus the expected value of future recourse actions. With a finite number of second stage realizations and all linear functions, we can always form the full deterministic equivalent linear program or extensive form. With many realizations, this form of the problem becomes quite large. The most common solution algorithm is the L-shape (Decomposition) method due to Van Slyke and Wets 1969, which solves two-stage SLP with $\omega$ having finite discrete distribution. For a brief discussion of SLP with recourse algorithms, see [1 chapter 7].

The basic difficulty of these methods is the need to perform too many iterations including solutions of many - quite large- linear programs which requires a powerful computer and more effort to reach a solution with small error.

\section{3- Application of the Two-stage SLP in Transportation problem:}


We consider a capacity expansion problem that has the form of a transportation problem. This example was contributed by David Morton at the University of Texas, [7]. A supply chain network with two suppliers is designed to supply three customers. The unit cost of supplying each customer from each supplier site is given in the following table:

\begin{tabular}{|c|c|c|c|}
\hline Transport Cost & Dem. 1 & Dem. 2 & Dem. 3 \\
\hline Supplier 1 & 4.3 & 2 & 0.5 \\
\hline Supplier 2 & 7.7 & 3 & 1 \\
\hline
\end{tabular}

The amounts required by the three demanders is uncertain. Each demand has three levels with amounts and probabilities given below. The probability distributions are independent.

\begin{tabular}{|c|cc|cc|cc|}
\hline $\begin{array}{c}\text { Demand/ } \\
\text { Probability }\end{array}$ & \multicolumn{2}{|c|}{ Dem. 1 } & \multicolumn{2}{c|}{ Dem. 2 } & \multicolumn{2}{c|}{ Dem. 3 } \\
\hline Level & $d_{1}$ & $P\left(d_{1}\right)$ & $d_{2}$ & $P\left(d_{2}\right)$ & $d_{3}$ & $P\left(d_{3}\right)$ \\
\hline 1 & 900 & 0.35 & 900 & 0.35 & 900 & 0.35 \\
\hline 2 & 1000 & 0.55 & 1000 & 0.55 & 1100 & 0.55 \\
\hline 3 & 1300 & 0.1 & 1250 & 0.1 & 1400 & 0.1 \\
\hline
\end{tabular}

The designer will install capacity at the two suppliers' sites. The first-stage decisions are $x_{1}$ and $x_{2}, 0$ the installed capacity at the two suppliers. The unit costs of installed capacity are $\$ 400$ and $\$ 350$ at supplier 1 and 2 respectively. The total capacity cannot exceed 10,000.

The reliability of the installed capacity is uncertain. The fractions $f_{1}$ and $f_{2}$ are the proportions of the installed capacity that will actually be available for satisfying demand. There are three levels of reliability as given in the table. These probability distributions are independent. They are also independent of the demand random variables.

\begin{tabular}{|c|cc|c|c|}
\hline $\begin{array}{c}\text { Reliability/ } \\
\text { Probability }\end{array}$ & \multicolumn{2}{|c|}{ Reliability 1} & \multicolumn{2}{c|}{ Reliability 2} \\
\hline Level & $f_{1}$ & $P\left(f_{1}\right)$ & $f_{2}$ & $P\left(f_{2}\right)$ \\
\hline 1 & 1 & 0.9 & 1 & 0.85 \\
\hline 2 & 0.95 & 0.05 & 0.8 & 0.1 \\
\hline 3 & 0.3 & 0.05 & 0 & 0.05 \\
\hline
\end{tabular}

The decision maker must select the supplier capacity to install before knowing the demand or reliability. The second-stage decisions are the decisions about which demands to service from the suppliers. In the following $y_{i j}$ is the demand satisfied at customer $j$ from supplier $i$. The problem has the form of a transportation model as shown below with random variables and decisions affecting the supplies and demands.

\begin{tabular}{|c|c|c|c|c|}
\hline Transport Cost & Dem. 1 & Dem. 2 & Dem. 3 & Supply \\
\hline Gen. 1 & 4.3 & 2 & 0.5 & $f_{1} x_{1}$ \\
\hline Gen. 2 & 7.7 & 3 & 1 & $f_{2} x_{2}$ \\
\hline Demand & $d_{1}$ & $d_{2}$ & $d_{3}$ & \\
\hline
\end{tabular}

The transportation model cannot be immediately solved because its parameters depend on five random variables and two decisions determined elsewhere. 
The two-stage SLP model of this problem is formulated as follows:

$$
\begin{aligned}
& \min z=c^{T} x+\mathcal{Q}(x) \\
& \text { s.t } x_{1}+x_{2} \leq 10000
\end{aligned}
$$

where the expected recourse cost is: $\mathcal{Q}(x)=E[Q(x, \xi)]$

$$
Q(x, \xi)=\min \sum_{i=1}^{2} \sum_{j=1}^{3} q_{i j} y_{i j}
$$

S.t.

$$
\begin{aligned}
& \sum_{j=1}^{3} y_{i j} \leq x_{i} f_{i} \quad i=1,2 \\
& \sum_{i=1}^{2} y_{i j} \geq d_{j} \quad j=1,2,3
\end{aligned}
$$

Here, the random vector $\xi \in R^{5}$, where $\xi=\left(f_{1}, f_{2}, d_{1}, d_{2}, d_{3}\right)$ and $P(\xi)=P\left(f_{1}\right) \cdot P\left(f_{2}\right) \cdot P\left(d_{1}\right) \cdot P\left(d_{2}\right) \cdot P\left(d_{3}\right)$ for each combination of the 5 random variables since all random variables are independent. The difficulty of using the L-shape algorithm is clear in this example. The two models can be combined to form a deterministic linear programming model that represents all 243 combinations of the random variables explicitly. This model has 2,189 decision variables and 1,216 structural constraints. After 13 optimality cuts and 7345 simplex iterations we obtain the solution $x=(2846,803)$ with total expected cost $1927689 \$$. However, this is not the optimum because a gap of 2.66 remains between the lower and upper bounds. A better solution with smaller tolerance can be obtained but it requires more iterations!!. In practical life, the problem may have more variables and more random variables with more realizations. Carrying on the L-shape algorithm in that case will be very difficult and the result will have low precision.

The major importance in this example is to compute the total expected cost with relatively small risk before knowing the realizations of the random variables in such a way that if the cost after realization deviates from the calculated, this deviation (risk) is affordable. For example, if this problem represents a power supply chain that a government wants to establish. It is a must to calculate the capacity to install at each power generator and the total expected cost with low penalty before starting the project. We studied four different treatments and compared the total cost in each case.

\section{Case 1: Design with subcontract:}

In this design, an additional subcontract (supply) is added to supply the demands not met by the original suppliers. In other words, if the demands exceed the amounts $\left(x_{1}, x_{2}\right)$ decided from the first stage, they are compensated from the subcontract but with relatively high cost. This can be considered as a penalty cost for not meeting the demands.

The problem is shown in the following table: 


\begin{tabular}{|l|l|l|l|c|}
\hline Transport Cost & Dem. 1 & Dem. 2 & Dem. 3 & Supply \\
\hline Gen. 1 & 4.3 & 2 & 0.5 & $f_{1} x_{1}$ \\
\hline Gen. 2 & 7.7 & 3 & 1 & $f_{2} x_{2}$ \\
\hline Subcontract & 6000 & 6000 & 6000 & no limit \\
\hline Demand & $d_{1}$ & $d_{2}$ & $d_{3}$ & \\
\hline
\end{tabular}

The recourse Model:

$$
\begin{gathered}
Z=\min 400 x_{1}+350 x_{2}+\mathcal{Q}(x) \\
x_{1}+x_{2} \leq 10000
\end{gathered}
$$

Where $\mathcal{Q}(x)=E_{\xi}[Q(x, \xi)]$, and:

$$
Q(x, \xi)=\min \sum_{i=1}^{3} \sum_{j=1}^{3} q_{i j} y_{i j}
$$

s.t.

$$
\begin{aligned}
& \text { supply constrains: } \sum_{j=1}^{3} y_{i j} \leq f_{i} x_{i} \quad i=1,2 \\
& \text { demand constrains: } \sum_{i=1}^{3} y_{i j} \geq d_{j} \quad j=1,2,3 \\
& \boldsymbol{X} \geq \mathbf{0}, \quad \boldsymbol{Y} \geq \mathbf{0}
\end{aligned}
$$

first, we solved the corresponding deterministic LP problem in case of substituting all random variables with their expected values. Then, we used the solution $\hat{X}$ to solve stage two for all combinations of the random variables. The total cost in this case is a random variable has 243 realizations. The same procedure is repeated but the random variables were replaced by their most likely values.

\section{Case2: Design with Extra-demand:}

In this design, instead of adding a subcontract to supply the over-demands, we design the suppliers as if they have to supply another extra-demand. This extra-demand represents the risk in case of over demands, but before the realization of the random variables. The problem is formulated in the following table:

\begin{tabular}{|l|l|l|l|l|c|}
\hline Transport Cost & Dem. 1 & Dem. 2 & Dem. 3 & Extra Dem & Supply \\
\hline Gen. 1 & 4.3 & 2 & 0.5 & 6000 & $f_{1} x_{1}$ \\
\hline Gen. 2 & 7.7 & 3 & 1 & 6000 & $f_{2} x_{2}$ \\
\hline Demand & $d_{1}$ & $d_{2}$ & $d_{3}$ & & \\
\hline
\end{tabular}

With the same procedure of case 1 and solving the second stage one time with the expectation and another time with the most likely values. The probability distributions of the total cost in each case are shown in the following figure:

Figure(1): pmf of total expected cost, case1 (using expectations) 


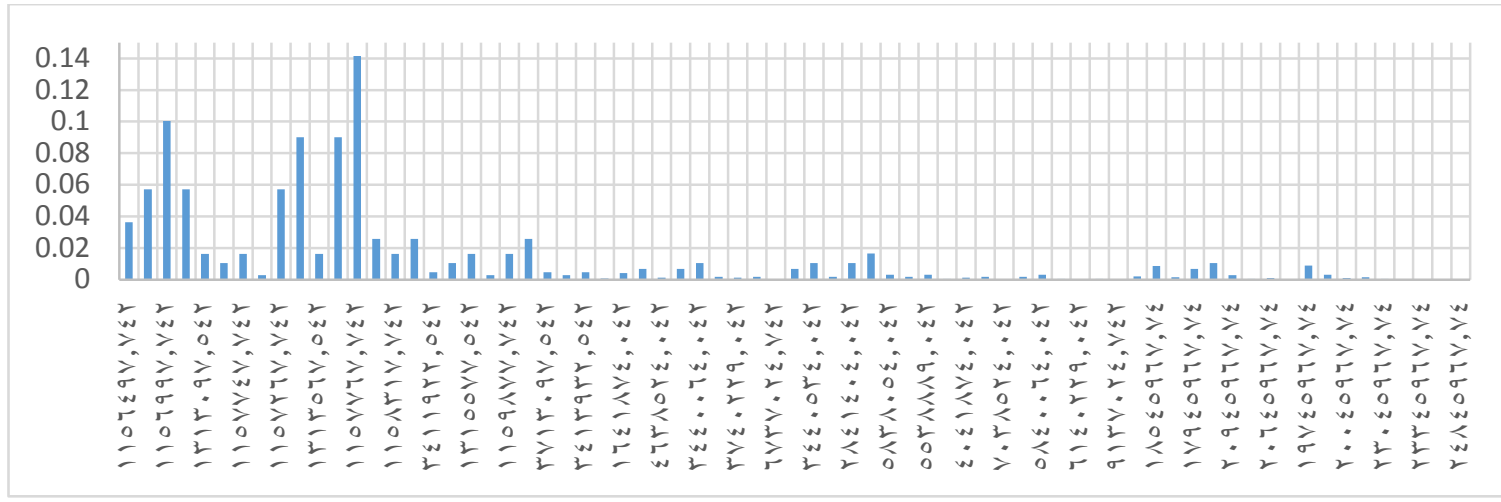

Figure(2): pmf ot total expected cost, case1 (using the most likely values)

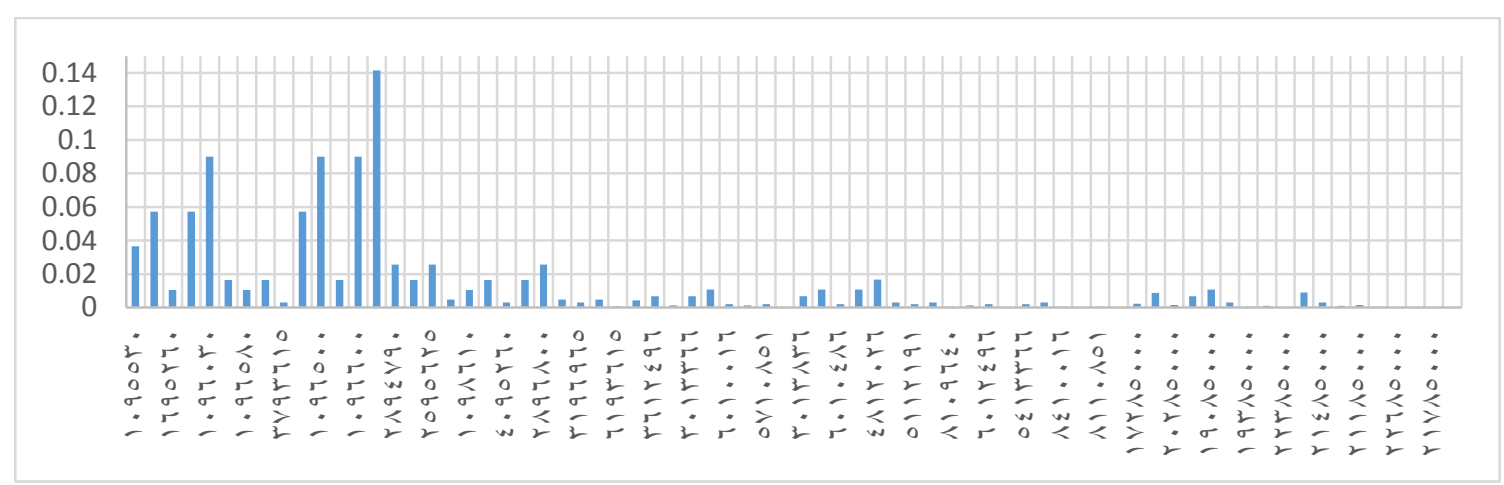

Figure(3): pmf of total expected cost, case2 (using expectations)

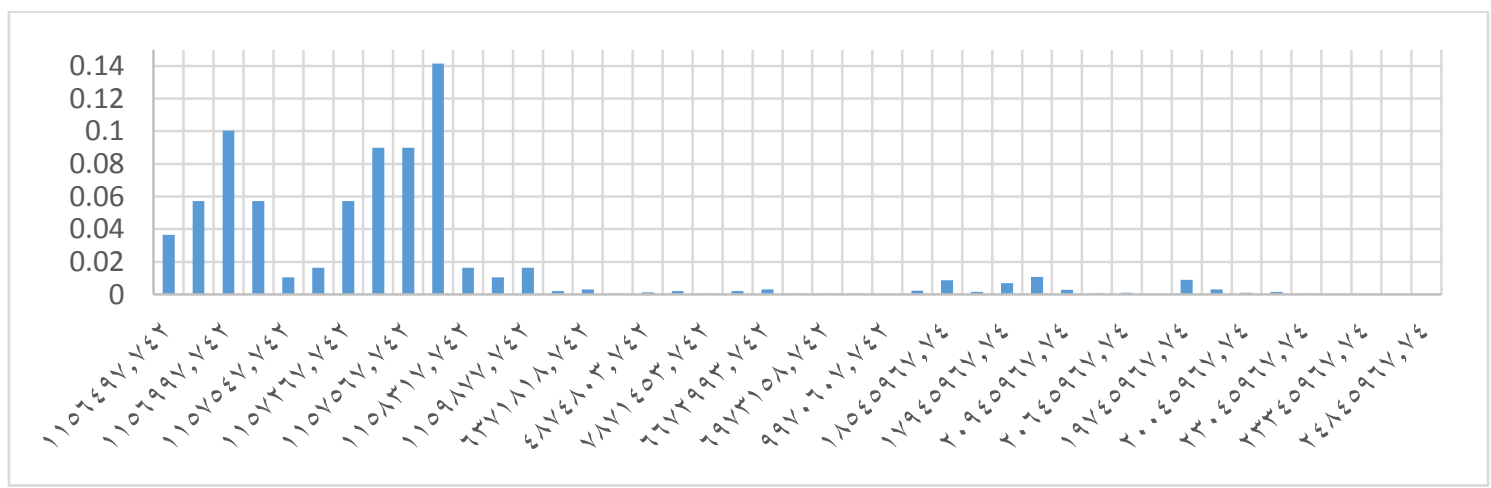

Figure(4): pmf of total expected cost, case2 (using the most likely values) 


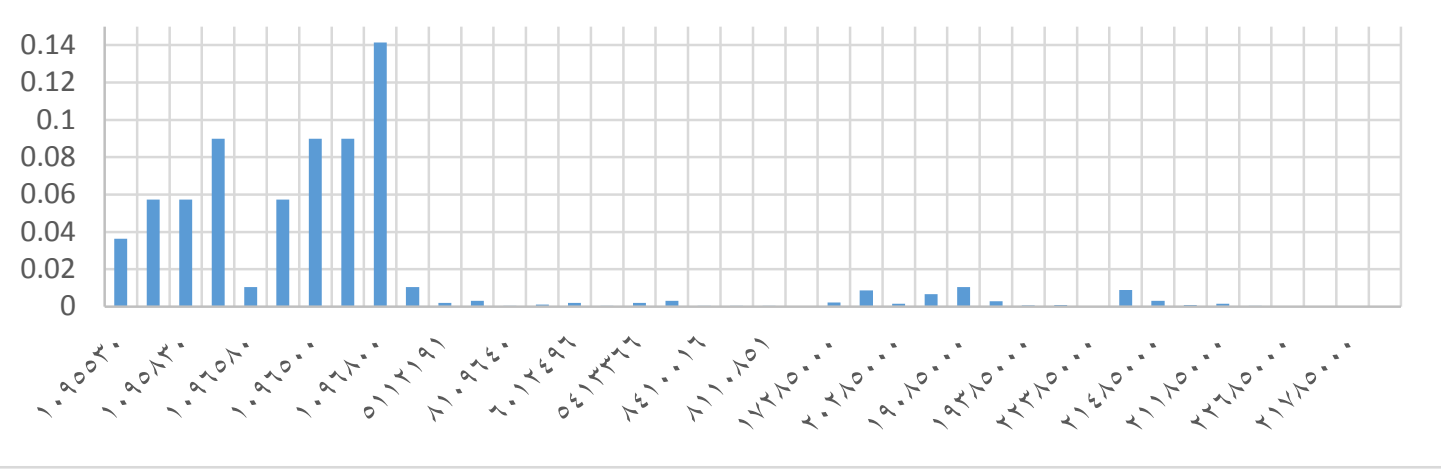

\section{Analysis of variance of the four treatments [6]}

Now, we have four distinct treatments for studying our example. ANOVA is used to compare a set of independent samples from a set of several populations. Since not all the samples are of equal size, then the data set is unbalanced. These type of sets are called one-factor layout, and have k-levels corresponding to $\mathrm{k}$ populations under testing. Our data set is a one-factor layout with 4-levels, the size of the data set is $n_{T}=792$. The ANOVA is based on the modeling assumption:

$x_{i j}=\mu_{i}+\epsilon_{i j}$ where $x_{i j}$ represents the $j t h$ observation from the ith population, and the error terms $\epsilon_{i j}$ are independently distributed as:

$$
\epsilon_{i j} \sim N\left(0, \sigma^{2}\right)
$$

Thus, $x_{i j}$ can be considered observation from a:

$$
N\left(\mu_{i}, \sigma^{2}\right)
$$

Point estimates of the unknown population means $\mu_{i}$ are obtained as the $k$ sample avareges. An assessment of the evidence that there is a difference between some of the population means can be made by testing the null hypothesis:

$$
H_{0}: \mu_{1}=\cdots .=\mu_{k}, \quad H_{A}: \mu_{i} \neq \mu_{j} \quad \text { for some } i \text { and } j
$$

Acceptance of the null hypothesis indicates that there is no evidence that any of the population means are unequal. Rejection of the null hypothesis implies that there is evidence that at least some of the population means are unequal, and therefore that it is not plausible to assume that the population means are all equal. In our example,

\begin{tabular}{|l|l|l|l|}
\hline Level $1 \boldsymbol{n}_{\mathbf{1}}=\mathbf{2 4 3}$ & Level $2 \boldsymbol{n}_{\mathbf{2}}=\mathbf{2 4 3}$ & Level $3 \boldsymbol{n}_{\mathbf{3}}=\mathbf{1 5 9}$ & Level $4 \boldsymbol{n}_{\mathbf{4}}=\mathbf{1 4 7}$ \\
\hline$\widehat{\boldsymbol{\mu}}_{\mathbf{1}}=\overline{\boldsymbol{X}}_{\mathbf{1}}=2434345$ & $\hat{\mu}_{2}=\bar{X}_{2}=2648750$ & $\hat{\mu}_{3}=\bar{X}_{3}=1878413$ & $\hat{\mu}_{4}=\bar{X}_{4}=1700852$ \\
\hline
\end{tabular}

Now, testing the hypothesis:

$$
\begin{aligned}
& H_{0}: \mu_{1}=\mu_{2}=\mu_{3}=\mu_{4} \\
& H_{A}: \mu_{i} \neq \mu_{j} \text { for some } i, j=1,2,3,4, \quad i \neq j
\end{aligned}
$$

The average of all the data observations is : $\bar{X}=2252379.99$ 
The some of squars for treatments $:$ SSTr $=2.69918 E+15$.

As the variability among the population mean estimates $\hat{\mu}_{i}=\bar{X}_{i}$ increases, the SSTr also increases. I.e. The sum of squares for treatments SSTr is a measure of the variability between the factor levels.

Another important consideration in the analysis of a one-factor layout is the amount of variability within the $k$ factor levels. This variability can be attributed to the variance $\sigma^{2}$ of the error terms $\epsilon_{i j}$ and can be measured with the statistic known as the sum of squares for error. $S S E=3.47651 E+16$.

The sum of squares for error SSE is a measure of the variability within the factor levels.

If the $k$ factor levels are ignored so that the data set is thought of as one big sample of size $n_{T}$, then the total variability of the total sample can be measured by SST (total sum of squares). $S S T=2.64868 E+16$

The total sum of squares SST is a measure of the total variability in the data set.

\section{P-value consideration:}

The plausibility of the null hypothesis that the factor level means are all equal depends upon the relative size of the sum of squares for treatments SSTr to the sum of squares for error SSE.

The p-value is directly proportional to the SSE and inversely proportional to the SSTr.

The SSTr in our example has 3 degrees of freedom, Mean squares of treatments

$$
\boldsymbol{M S T r}=\frac{S S T r}{K-1}=8.99727 E+14
$$

The SSE has $n_{T}-k=788$ degrees of freedom, and the Mean square error MSE

$$
M S E=\frac{S S E}{n_{T}-k}=4.41182 E+13 .
$$

The MSE is an unbiased point estimate of the error variance $\sigma^{2}$, since $E(M S E)=\sigma^{2}$. Consequently, MSE is sometimes written as $\hat{\sigma}^{2}$.

To calculate the p-value for the null hypothesis:

$$
H_{0}: \mu_{1}=\mu_{2}=\mu_{3}=\mu_{4}
$$

When this null hypothesis is true, then the F-statistics:

$$
F=\frac{M S T r}{M S E} \sim F_{k-1, n_{T}-k}
$$

The plausibility of the null hypothesis is therefore in doubt whenever the observed value of the $F$-statistic does not look like it is an observation from an $F_{k-1, n_{T}-k}$ distribution. 
Moreover, MSTr has a larger expected value when the factor means $\mu_{i}$ are unequal than under the null hypothesis, thus, larger values of the F-statistics cast doubt on the plausibility of the null hypothesis. Consequently, the p-value is calculated as:

$$
p-\text { value }=P(X \geq F)
$$

Where the random variable $X$ has an $F_{k-1, n_{T}-k}$ distribution.

In our example $F=\frac{M S T r}{M S E}=20.39355$ and $X$ has an $F_{3,788}$ distribution, then:

$$
p-\text { value }=P(X \geq 20.39355) \cong 0
$$

The ANOVA table for our example is given:

\begin{tabular}{|l|l|l|l|l|l|}
\hline Sources & Degrees of freedom & Sum of squares & Mean squares & F-statistics & p-value \\
\hline Treatments & 3 & $2.69918 \mathrm{E}+15$ & $8.99727 \mathrm{E}+14$ & 20.39355 & 0.00 \\
\hline Error & 788 & $3.47651 \mathrm{E}+16$ & $4.41182 \mathrm{E}+13$. & & \\
\hline total & 791 & $2.64868 \mathrm{E}+16$ & & & \\
\hline
\end{tabular}

Thus the null hypothesis is not plausible.

Since the null hypothesis is rejected, we shall follow up the analysis with pairwise comparisons of the factor level means to discover which ones have been shown to be different and by how much. With 4 factor levels we have 6 pairwise differences.

These confidence intervals are often referred to as the Tukey multiple comparisons procedure. Inb our example we calculated $95 \%$ confidence level simultaneous confidence

\begin{tabular}{|c|c|c|c|c|}
\hline $\mathbf{n}$ & $\begin{array}{l}\text { Pairwise } \\
\text { diff. }\end{array}$ & Mean and size & & Confidence interval \\
\hline \multirow[t]{2}{*}{1} & \multirow[t]{2}{*}{$\mu_{1}-\mu_{2}$} & $\bar{X}_{1}=2434345$ & $n_{1}=243$ & \multirow[t]{2}{*}{$(-1761127.381,1332317.381)$} \\
\hline & & $\bar{X}_{2}=2648750$ & $n_{2}=243$ & \\
\hline \multirow[t]{2}{*}{2} & \multirow[t]{2}{*}{$\mu_{1}-\mu_{3}$} & $\bar{X}_{1}=2434345$ & $n_{1}=243$ & \multirow[t]{2}{*}{$(-1183117.07,2294981)$} \\
\hline & & $\bar{X}_{3}=1878413$ & $n_{3}=159$ & \\
\hline \multirow[t]{2}{*}{3} & \multirow[t]{2}{*}{$\mu_{1}-\mu_{4}$} & $\bar{X}_{1}=2434345$ & $n_{1}=243$ & \multirow[t]{2}{*}{$(-1047946.215,2514932.21)$} \\
\hline & & $\bar{X}_{4}=1700852$ & $n_{4}=147$ & \\
\hline \multirow[t]{2}{*}{4} & \multirow[t]{2}{*}{$\mu_{2}-\mu_{3}$} & $\bar{X}_{2}=2648750$ & $n_{2}=243$ & \multirow[t]{2}{*}{$(-968712.07,2509386.07)$} \\
\hline & & $\bar{X}_{3}=1878413$ & $n_{3}=159$ & \\
\hline \multirow[t]{2}{*}{5} & \multirow[t]{2}{*}{$\mu_{2}-\mu_{4}$} & $\bar{X}_{2}=2648750$ & $n_{2}=243$ & \multirow[t]{2}{*}{$(-833541.215,2729337.215)$} \\
\hline & & $\bar{X}_{4}=1700852$ & $n_{4}=147$ & \\
\hline
\end{tabular}
intervals: 


\begin{tabular}{|l|l|l|l|l|}
\hline $\mathbf{6}$ & $\boldsymbol{\mu}_{\mathbf{3}}-\boldsymbol{\mu}_{\mathbf{4}}$ & $\bar{X}_{3}=1878413$ & $n_{3}=159$ & \multirow{2}{*}{$(-1773199.677,2128321.677)$} \\
\cline { 3 - 4 } & & $\bar{X}_{4}=1700852$ & $n_{4}=147$ & \\
\hline
\end{tabular}

Since intervals 1 to 6 contain 0 , then there is no evidence that the means of factor levels pairs are different.

The following graph shows by how much the factor levels means are different.

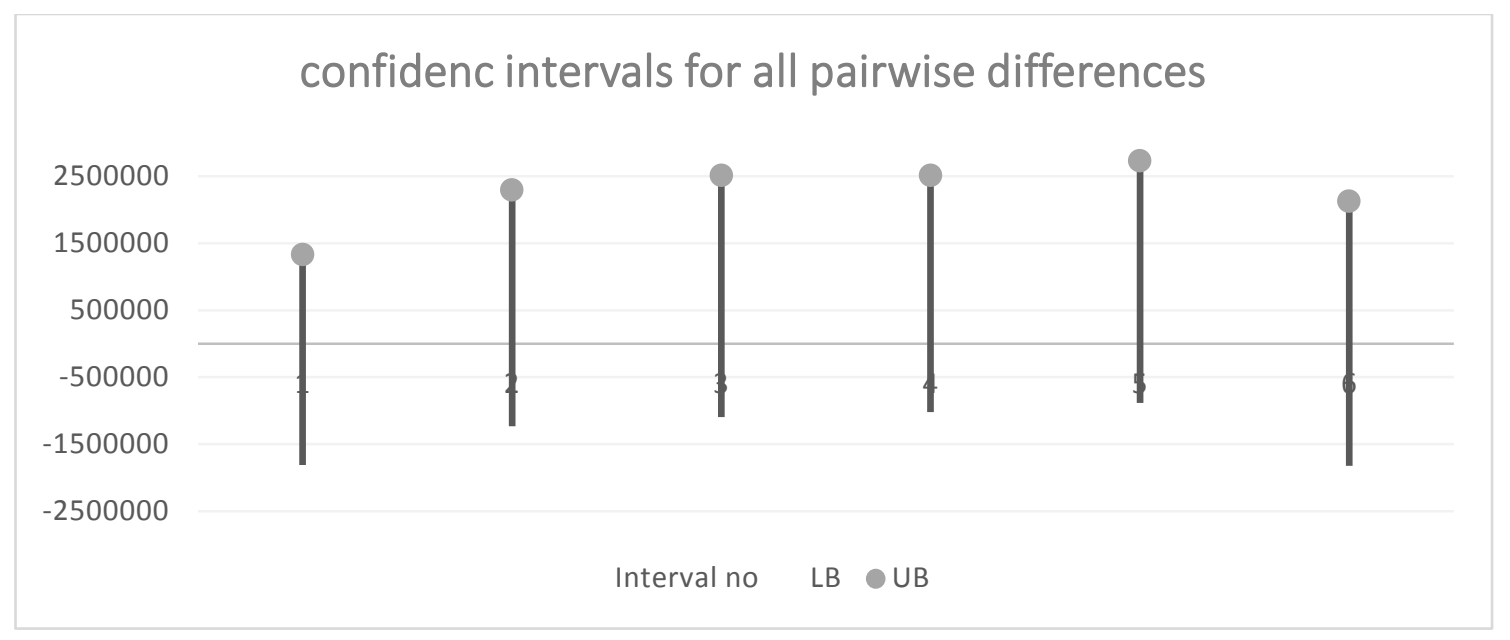

\section{3- Conclusion:}

According to the results obtained from the previous numerical studies, the following conclusions can be made. There is no evidence that any two population means of the four treatments are different, therefore, it is plausible to assume that all the population means are equal. Also, there is no evidence that designing with subcontract and using either the expected values or the most likely values of the random variables will give, in average, quite different total expected costs. The confidence interval of the difference between the means of both of them shows a small variability between them. There is no evidence that designing with extra demand and using either the expectation or the most likely values will give, in average, different total expected cost, but the variability between them is relatively high.

\section{References}

[1] Peter Kall, Janson Mayer, "Stochastic Linear Programming Models, Theory, and Computation", Springer's international series, 2005.

[2] John R. Birge, Franc sois Louveaux, " Introduction to Stochastic Programming”, second edition, Springer series in operation research,2011.

[3] Alexander Shapiro, Darinka Dentcheva, Andrzej Ruszczynski, " Lectures on Stochastic Programming Modeling And Theory", SIAM, 2009.

[4] Suvrajeet sen, Julia L Higle, "An Introductory tutorial on Stochastic Linear Programming Models", Institute for Operations Research and the Management Sciences, pp. 33-61, March 1999.

[5] Singiresu S. Rao, “Engineering Optimization Theory and Practic”, John Wiley \& Sons, INC., fourth edition, 2009. 
[6] Anthony Hayter," Probability and Statistics for Engineers and Scientists", Brooks/Cole, Cengage Learning, fourth edition, 2012.

[7] Paul A. Jensen, Jonathan F. Bard, “ Operation Research Models and Methods”, John Wiley \& Sons, INC.,2003. 